Original Article

\title{
The Effect of Resveratrol Supplementation in Adjunct with Non-surgical Periodontal Treatment on Blood Glucose, Triglyceride, Periodontal Status and Some Inflammatory Markers in Type 2 Diabetic Patients with Periodontal Disease
}

\author{
Ahmad Zare Javid ${ }^{1}$, Razie Hormoznejad*2 ${ }^{2}$, Hojat allah Yousefimanesh ${ }^{3}$, Mehrnoosh Zakerkish ${ }^{4}$, Mohammad Hosein Haghighi-zadeh ${ }^{5}$ \\ Ali Barzegar ${ }^{6}$, Nasim Niknejad ${ }^{2}$ \\ 1- Nutrition and Metabolic Diseases Research Center \& Hyperlipidemia Research Center, Ahvaz Jundishapur University of Medical Sciences, Ahvaz, \\ Iran. \\ 2- Student Rresearch Committee, School of Paramedicine, Ahvaz Jundishapur University of Medical Science, Ahvaz, Iran. \\ 3- Dept.of Periodontology, School of Dentistry, Ahvaz Jundishapur University of Medical Sciences, Ahvaz, Iran. \\ 4- Health research institute, Diabetes research center, Ahvaz Jundishapur University of Medical Sciences, Ahvaz, Iran \\ 5- School of Public Health, Ahvaz Jundishapur University of Medical Sciences, Ahvaz, Iran. \\ 6- Dept.of Community Nutrition, Faculty of Nutrition, Tabriz University of Medical Sciences, Tabriz, Iran
}

\section{A B S T R A C T}

Background and Objectives: Diabetes mellitus and periodontal disease are two chronic and common diseases with close relationship together affecting public health and quality of life. The aim of this study was to investigate the effect of resveratrol supplementation in adjunct with non-surgical periodontal treatment (NST) on blood glucose, triglyceride, periodontal status and inflammatory markers in type 2 diabetic patients with periodontal disease.

Materials and Methods: This double-blind clinical trial study was conducted on 43 diabetic patients with periodontitis referred to the Endocrinology Clinic at Golestan Hospital in Ahvaz, Iran. All subjects were randomly assigned into two groups of intervention and control. The intervention and control groups received either $480 \mathrm{mg} / \mathrm{d}$ resveratrol or placebo capsules (2 PCs) for four weeks. All subjects underwent non-surgical periodontal therapy during the intervention period. Anthropometric parameters, 24-hour dietary recall, fasting blood sugar, insulin, insulin resistance (HOMA-IR), triglycerides, pocket depth (PD), IL6 and TNF $\alpha$ were evaluated in all subjects pre- and post-intervention.

Results: The mean serum levels of fasting insulin and insulin resistance (HOMA-IR) were significantly $(\mathrm{P}=0.02, \mathrm{P}=0.045$, respectively) lower in the intervention group compared with the control group $(10.42 \pm 0.28$ and $10.92 \pm 0.9 ; 3.66 \pm 0.97$ and $4.49 \pm 1.56$, respectively $)$. Moreover, significant difference $(\mathrm{P}<0.001)$ was obtained in the mean pocket depth (PD) between the intervention and control groups ( $2.35 \pm 0.6$ and $3.38 \pm 0.5$, respectively) post-intervention. In the intervention group, the mean serum level of IL6 was reduced significantly $(\mathrm{P}=0.039)$ post-intervention $(1.58 \pm 1.06$ and $2.19 \pm 1.09)$. No significant differences were seen in the mean levels of fasting blood sugar, triglycerides, IL6 and TNF $\alpha$ between the two groups post-intervention.

Conclusions: It is suggested that resveratrol may be recommended as adjuvant therapy along with non-surgical periodontal treatment in diabetic patients with periodontal disease.

Keywords: Type 2 diabetes mellitus, Periodontal disease, Resveratrol, Insulin resistance, Inflammatory markers

\section{Introduction}

Diabetes mellitus and periodontal disease are known as two global common and chronic diseases
(1). According to the World Health Organization (WHO) statistics, the prevalence of diabetes mellitus 
was about 382 million people in the world in 2013, reaching 438 million in $2030(2,3)$. On the other hand, it is indicated that more than $2 / 3$ of the world population are suffering from periodontal disease (4). There is a two-way relationship between these two diseases so that diabetes mellitus may increase the risk of periodontal disease and vice versa (5). It was reported that the prevalence of severe periodontitis is approximately 39 to $59.6 \%$ higher in diabetic than non-diabetic patients (6). The risk of periodontal disease is three times greater in diabetic subjects than in non-diabetic subjects $(7,8)$. Periodontal disease is a chronic inflammatory and infectious disease, affecting tissues supporting teeth, and is characterized by gingival bleeding, pocket formation, alveolar bone destruction, connective tissue degradation, and tooth loss $(9,10)$. Periodontal disease increases insulin resistance, and thereby may disturb glycemic control (11). It has been found that controlling blood sugar is six times lower in diabetic patients with periodontitis than in diabetic patients without periodontitis (12). It has also been indicated that type 2 diabetic patients with periodontal disease have higher levels of inflammatory markers such as IL-1 $\beta$, TNF $\alpha$ and IL-6. These inflammatory factors may adversely alter blood glucose and lipid metabolism (13). In a large cohort study in Taiwan (from 2003 to 2009), a significant association was observed between metabolic syndrome and diagnosis of periodontal disease (14). According to the findings of some studies, periodontal inflammation is associated with a significant increase in Hemoglobin A1c (HbA1c), Fasting Blood Sugar (FBS) and inflammatory factors in patients with diabetes mellitus $(15,16)$. Gram-negative anaerobic bacteria are thought to be the primary factor in the development of periodontal disease (17). Several researchers have investigated the beneficial effects of non-surgical periodontal therapy including oral health education, use of antibiotics, scaling and root planning (18). Mouthwash antibiotics are widely used to control periodontal disease; however, excessive use of antibiotics may lead to bacterial resistance against them. Therefore, a new treatment strategy is needed. Natural ingredients derived from plants such as polyphenols are widely considered for the treatment of periodontal disease (19). In addition, the importance of dietary factors in periodontal disease has been identified (20). Intake of nutrients with antioxidant properties along with other treatments in periodontal disease can play a useful role in coping with the oxidative stress leading to injured oral tissues including teeth (4). Resveratrol (3, 5, 4'trihydroxystilbene, a composition of phytoalexin) is produced by polyphenol plants of Stilbene in response to stressful irritations such as fungal and bacterial infections. Polygonum cuspidatum (the richest source of resveratrol) is a root extract, and is known as a herbal medicine. Resveratrol exists in foods such as grapes, peanuts, pistachio and cranberry $(21,22)$. Resveratrol is known as an anti-microbial (23), antiinflammatory, anti-apoptotic (24), anti-cancer substance and osteogenic factor (25). Resveratrol may have an antioxidant effect against oxidative stress in some chronic diseases such as diabetes mellitus and cardiovascular diseases $(24,26,27)$. It is assumed that in patients with periodontal disease, there may be a resistance to antibiotics; resveratrol with a strong antimicrobial and anti-inflammatory properties can play an important role in the treatment process. On the other hand, resveratrol is known as a French paradox, and may lower the mortality rate caused by cardiovascular diseases (28). Despite a higher annual sale of resveratrol supplement in the United States, there are only few human studies about its beneficial effects (29). Based on the results of the limited human and animal studies carried out on diabetic subjects, it was reported that resveratrol can reduce insulin resistance, plasma glucose, $\mathrm{HbA1c}$, FBS, and inflammatory factors; it may also improve insulin sensitivity $(24,27$, and 30$)$. There are many studies on the effects of non-surgical periodontal treatment on the recovery of metabolic status in these patients. Also there are only few studies investigating the impact of nutrition including the protecting effects of resveratrol on periodontal disease $(17,19,26$, and $31)$. In the case of the effectiveness, nutritional therapy can be used as a safest approach to prevent and treat periodontal disease. Therefore, the present study aimed to evaluate the effect of resveratrol supplementation in adjunct with non-surgical treatment of periodontal status on FBS, insulin, insulin resistance (HOMA-IR), triglycerides, pocket 
depth (PD) and inflammatory markers comprising IL6 and TNF $\alpha$ in diabetic patients with periodontal disease.

\section{Materials and Methods}

Samples and study design: This randomized doubleblind, placebo-controlled, clinical trial was conducted in Ahvaz Golestan Hospital, Iran. The diabetic patients referring to the Endocrinology Clinic with some symptoms of periodontal disease were referred to Dental Clinic for further diagnosis. The Flowchart for this study is shown in Fig. 1. With a power of $95 \%$, the total number of required subjects for this study was 40 people. The Homa-IR (24) was used for sample size calculation. Regarding with the inclusion and exclusion criteria, 50 patients (36 females and 14 males) were recruited. The subjects were randomly allocated into two groups of intervention $(n=25)$ and control $(n=25)$. Inclusion criteria included having age between 30 and 60 years, body mass index 18.5$30 \mathrm{~kg} / \mathrm{m}^{2}$, confirmed diabetes mellitus (no more than five years since diagnosis), FBS lower than $22 \mathrm{mmol} / \mathrm{L}$, and moderate periodontal disease. Exclusion criteria included suffering from diabetes mellitus complications such as kidney failure, pregnancy, breastfeeding, traveling more than two weeks, smoking, using immunosuppressive medications, using insulin, periodontal treatment history during the past six months, using any antioxidants, and considerable changes in diet over the past six months. This study was approved by the Ethics Committee of the Research Deputy of Ahvaz Jundishapur University of Medical Sciences (reference No. UR1393.364ajums.rec). A signed consent form was also obtained from all subjects at the beginning of the study. The intervention and control groups received either $480 \mathrm{mg} / \mathrm{d}$ resveratrol or placebo capsules ( 2 pills) for four weeks. Resveratrol capsules (480 mg Polygonum Cuspidatum providing $240 \mathrm{mg}$ resveratrol) were purchased from Herbafit. Placebo capsules contained $480 \mathrm{mg}$ starch. All subjects were monitored and contacted three times a week by telephone to ensure that they were using the prescribed capsules. During the study, all subjects used any usual blood glucose-lowering medications prescribed already. Moreover, all subjects underwent the non-surgical treatment (including removal of dental plaque, scaling and root planning) for periodontal disease. In addition, some instructions for dental hygiene (such as how to brush and use dental floss and mouthwash correctly) were provided to all subjects.

Anthropometric and nutritional assessments: Anthropometric indices including height, weight and waist-hip ratio were measured at the beginning and end of the study. Weight was measured by a Seca scale (Germany) to the nearest of $0.1 \mathrm{~kg}$, and height was measured using a stadiometer to the nearest of $0.1 \mathrm{~cm}$. Waist circumference (widest area between the edge of lower rib and iliac corset) and hip circumference (the largest pelvic girth) were measured to the nearest of $0.1 \mathrm{~cm}$. A 24-hour dietary recall was also obtained for dietary assessment at the beginning and end of the study.

Biochemical measurements: A fasting venous blood sample (5 ml) was obtained from all subjects pre- and post-intervention. All samples were centrifuged, and the serum samples were maintained in $-70^{\circ} \mathrm{C}$ freezer until the day of analysis except some samples for analyzing FBS. Serum levels of FBS, insulin, insulin resistance (HOMAIR), triglyceride (TG), IL6 and TNF $\alpha$ were evaluated. FBS was immedietly measured by enzymatic method using laboratory kits (Pars Azmoon, Tehran, Iran) and an auto-unalyzer. Serum level of insulin was determined by ELISA method using laboratory kit (Monobind ,USA). TG was also measured by colorimetric method using the laboratory kits of Pars Azmoon (Tehran, Iran). IL6 and TNFa were measured by ELISA method using laboratory kits (eBioscience, Germany). Insulin resistance (HOMA-IR) was calculated by the following formula:

HOMA-IR =Fasting glucose $(\mathrm{mmol} / \mathrm{L}) \times$ fasting insulin $(\mu \mathrm{U} / \mathrm{mL}) / 22.5$

Evaluation of periodontal status: Probing depth (PD) was measured by a single, calibrated examiner at 6 sites per tooth: mesiobuccal, midbuccal, distobuccal, mesiolingual, midlingual and distolingual sites. The PD was recorded using a UNC-15 (University of North Carolina No. 15) manual periodontal probe. Full mouth clinical measurements were conducted at baseline. Ten selected sites with the probing depth $\geq 4 \mathrm{~mm}$ were randomly selected 
from at least 3 of the quadrants for the clinical measurements after intervention.

Statistical analysis: Statistical analysis was done using SPSS (version 19). Data were reported as mean $\pm \mathrm{SD}$. In order to determine whether the data have a normal distribution, a visual examination of the data and a goodness of fit test (Kolmorogov-Smirnoff) were conducted. Independent sample t-test was used to compare the results between the two groups. Paired t-test was also used to compare the results within groups pre- and post-intervention. The confidence interval and power were considered at $95 \%$ and $95 \%$, respectively. P-values lower than 0.05 were considered significant.

\section{Results}

Totally, 50 patients were recruited of which 43 $(n=22$ in the control group and $n=21$ in the intervention group) completed the study. Seven patients did not use capsules regularly and were excluded. General and demographic characteristics of the subjects are shown in Table 1. Mean age of the subjects in the intervention and control groups was $49 \pm 7$ and $50 \pm 8$ years, and mean BMI of $29 \pm 4.9$ and $28 \pm 4.8 \mathrm{~kg} / \mathrm{m}^{2}$, respectively. There was no significant difference between the two groups in mean age, BMI, gender ratio, weight, waist circumference, hip circumference, FBS, fasting insulin, insulin resistance (HOMA-IR), TG, IL6 and TNF $\alpha$ at baseline. No significant differences were also seen between the two groups for dietary data including the intakes of energy, macronutrients and micronutrients such as antioxidant vitamins $\mathrm{C}, \mathrm{E}$, and $\mathrm{A}, \alpha$-tocopherol and selenium at baseline and after the intervention (Table 2).

Table 1: Baseline characteristics of the subjects

\begin{tabular}{|c|c|c|c|}
\hline Varieble & $\begin{array}{l}\text { Control group } \\
(n=22)\end{array}$ & $\begin{array}{l}\text { Intervention group } \\
(\mathrm{n}=21)\end{array}$ & $P$-value \\
\hline Age (years) & $50.9 \pm 8.9$ & $49.1 \pm 7.4$ & 0.48 \\
\hline $\begin{array}{l}\text { Gender } \\
\qquad \begin{array}{l}\text { Female }(\mathrm{N}) \\
\text { Male }(\mathrm{N})\end{array}\end{array}$ & $\begin{array}{l}18 \\
4\end{array}$ & $\begin{array}{l}16 \\
5\end{array}$ & $0.72^{\dagger}$ \\
\hline Weight $(\mathrm{kg})$ & $70.95 \pm 11$ & $73.8 \pm 10.2$ & 0.38 \\
\hline Height (m) & $1.58 \pm 0.05$ & $1.59 \pm 0.09$ & 0.8 \\
\hline BMI (kg/m2) & $28.3 \pm 4.8$ & $29.3 \pm 4.9$ & 0.48 \\
\hline Waist circumference $(\mathrm{cm})$ & $100 \pm 8.9$ & $101.4 \pm 8.8$ & 0.61 \\
\hline Hip circumference (cm) & $107.5 \pm 9.5$ & $107.8 \pm 8.1$ & 0.92 \\
\hline Fasting blood glucose (mmol/l) & $9.4 \pm 3$ & $8.5 \pm 3$ & 0.34 \\
\hline Insulin $(\mu \mathrm{u} / \mathrm{ml})$ & $11.2 \pm 1.3$ & $11.3 \pm 1.7$ & 0.85 \\
\hline Homa-ir & $4.6 \pm 1.3$ & $4.2 \pm 1.4$ & 0.32 \\
\hline Triglyceride $(\mathrm{mg} / \mathrm{dL})$ & $137.7 \pm 66$ & $147 \pm 69$ & 0.65 \\
\hline IL-6 ( pg/ml) & $2.08 \pm 0.8$ & $2.19 \pm 1$ & 0.71 \\
\hline TNF- $\alpha(\mathrm{pg} / \mathrm{ml})$ & $10.56 \pm 0.6$ & $10.49 \pm 0.47$ & 0.6 \\
\hline $\mathrm{PD}(\mathrm{mm})$ & $4.06 \pm 0.6$ & $3.54 \pm 0.5$ & 0.29 \\
\hline
\end{tabular}


Ahmad Zare Javid, et al: Resveratrol supplementation in diabetic patients with periodontitis

Table 2. Mean \pm SD of energy, macronutrients and micronutrients intake

\begin{tabular}{|c|c|c|c|c|}
\hline Variable & & Baseline & After 4 weeks & $P$-value \\
\hline \multirow[t]{3}{*}{ Energy (kcal/d) } & Control group & $1812.85 \pm 329.7$ & $1803.2 \pm 265.6$ & 0.85 \\
\hline & Intervention group & $1819.81 \pm 312.3$ & $1838.54 \pm 321.9$ & 0.8 \\
\hline & $P$-value ${ }^{*}$ & 0.94 & 0.69 & \\
\hline \multirow[t]{3}{*}{ Carbohydrate (g/d) } & Control group & $238.72 \pm 55.6$ & $233.17 \pm 49.9$ & 0.5 \\
\hline & Intervention group & $249.86 \pm 60.5$ & $251.5 \pm 66$ & 0.91 \\
\hline & $P$-value & 0.53 & 0.3 & \\
\hline \multirow[t]{3}{*}{ Protein (g/d) } & Control group & $70.48 \pm 29.37$ & $72.7 \pm 24.39$ & 0.48 \\
\hline & Intervention group & $61.4 \pm 21.3$ & $63.1 \pm 19$ & 0.72 \\
\hline & $P$-value & 0.25 & 0.15 & \\
\hline \multirow[t]{3}{*}{ Fat $(g / d)$} & Control group & $65.82 \pm 19.14$ & $65.52 \pm 20.43$ & 0.9 \\
\hline & Intervention group & $66.46 \pm 24.2$ & $66.84 \pm 16.9$ & 0.94 \\
\hline & $P$-value & 0.92 & 0.82 & \\
\hline \multirow[t]{3}{*}{ Selenium (mg/d) } & Control group & $0.056 \pm 0.047$ & $0.056 \pm 0.033$ & 0.97 \\
\hline & Intervention group & $0.076 \pm 0.043$ & $0.07 \pm 0.04$ & 0.62 \\
\hline & $P$-value & 0.14 & 0.2 & \\
\hline \multirow[t]{3}{*}{ Vitamin A (mcg/d) } & Control group & $287.9 \pm 348.3$ & $335.36 \pm 683.8$ & 0.78 \\
\hline & Intervention group & $562.05 \pm 1397.8$ & $256.56 \pm 235.6$ & 0.25 \\
\hline & $P$-value & 0.37 & 0.62 & \\
\hline \multirow[t]{3}{*}{ Beta-Carotene $(\mathrm{mcg} / \mathrm{d})$} & Control group & $50.9 \pm 142$ & $19.7 \pm 18$ & 0.7 \\
\hline & Intervention group & $55.4 \pm 59.37$ & $58.1 \pm 56.9$ & 0.38 \\
\hline & $P$-value & 0.89 & 0.7 & \\
\hline \multirow[t]{3}{*}{ Vitamin C (mg/d) } & Control group & $101.5 \pm 106.9$ & $82.9 \pm 108.2$ & 0.19 \\
\hline & Intervention group & $89.73 \pm 81.8$ & $87.8 \pm 67.6$ & 0.92 \\
\hline & $P$-value & 0.68 & 0.85 & \\
\hline \multirow[t]{3}{*}{ Vitamin E (mg/d) } & Control group & 2.83 & 2.48 & 0.67 \\
\hline & Intervention group & 2.93 & 2.72 & 0.56 \\
\hline & $P$-value & 0.9 & 0.6 & \\
\hline \multirow[t]{3}{*}{$\alpha$-tocopherol (mg/d) } & Control group & $7.29 \pm 3.21$ & $7.09 \pm 3.06$ & 0.75 \\
\hline & Intervention group & $6.67 \pm 4.1$ & $7.31 \pm 2.82$ & 0.38 \\
\hline & $P$-value & 0.58 & 0.8 & \\
\hline
\end{tabular}

$* \mathrm{P}<0.05$ was considered as significant using Independent $\mathrm{T}$-test between the two groups at baseline and post-intervention $* * \mathrm{P}<0.05$ was considered as significant using Paired T-test.

Table 3 shows the biochemical parameters pre- and post-intervention in the two groups. Although the mean FBS was higher in the intervention group compared with the control group $(7.9 \pm 2.12$ and 9.19 \pm 2.78$)$ post-intervention, it was not significant $(\mathrm{P}=0.097)$. Furthermore, the mean FBS was reduced (but not significantly) in the intervention group postintervention $(8.51 \pm 3.13$ and $7.9 \pm 2.12 ; \quad \mathrm{P}=0.23)$. Regarding with the serum levels of fasting insulin, There was a significant difference $(\mathrm{P}=0.02)$ between the intervention and control groups $(10.42 \pm 0.28$ and $10.92 \pm 0.9$ respectively) post-intervention. Moreover, insulin level was significantly $(\mathrm{P}=0.03)$ reduced in the intervention group post-intervention compared to the baseline (10.42 \pm 0.28 and $11.32 \pm 1.76$, respectively). The mean HOMA-IR was significantly $(\mathrm{P}=0.045)$ lower in the intervention group compared with the control group (3.66 \pm 0.97 and $4.49 \pm 1.56$, respectively) post-intervention. Furthermore, within group comparison in the intervention group showed that the mean HOMA-IR was reduced significantly $(\mathrm{P}=0.025)$ post-intervention compared with the baseline (3.66 \pm 0.97 and $4.22 \pm 1.43$, respectively); however, this was not significant in the control group. There was no significant difference in TG level between the intervention and control groups post-intervention $(135 \pm 60.89$ and $147 \pm 69.3 \mathrm{mg} / \mathrm{dL}, \mathrm{p}=0.65)$. Moreover, no significant $(\mathrm{P}=0.3)$ difference was obtained in the mean level of IL6 between the two groups $(1.58 \pm 1.06$ and $1.85 \pm 0.59$, respectively) post-intervention. However, in the intervention group, the mean level of IL6 was significantly $(\mathrm{P}=0.039)$ reduced post- 
intervention compared with baseline $(1.58 \pm 1.06$ and $2.19 \pm 1.09$, respectively). Furthermore, the mean serum level of $\mathrm{TNF} \alpha$ did not show a significant $(\mathrm{P}=0.25)$ difference betweenthe intervention and control groups $(10.33 \pm 0.66$ and $10.57 \pm 0.68$, respectively) post-intervention. Regarding with pocket depth (PD), there was a significant difference $(\mathrm{P}<0.001)$ between the two groups $(2.35 \pm 0.6$ and $3.38 \pm 0.5 \mathrm{~mm}$, respectively) postintervention. Moreover, PD was significantly reduced in the intervention and control groups after 4 weeks compared to the baseline (Table 4).

Table 3. Biochemical variables at baseline and post-intervention.

\begin{tabular}{|c|c|c|c|c|c|c|c|}
\hline \multirow[b]{2}{*}{ Variable } & \multicolumn{3}{|c|}{ Control group $(n=22)$} & \multicolumn{3}{|c|}{ Intervention group $(n=21)$} & \multirow[t]{2}{*}{$P$-value **: } \\
\hline & Baseline & After 4 weeks & $P$-value $*$ & Baseline & After 4 weeks & $P$-value * & \\
\hline Fasting blood glucose $(\mathrm{mmol} / \mathrm{l})$ & $9.42 \pm 3.02$ & $9.19 \pm 2.78$ & 0.64 & $8.51 \pm 3.13$ & $7.9 \pm 2.12$ & 0.23 & 0.097 \\
\hline Insulin $(\mu \mathrm{u} / \mathrm{ml})$ & $11.23 \pm 1.35$ & $10.92 \pm 0.9$ & 0.21 & $11.32 \pm 1.76$ & $10.42 \pm 0.28$ & 0.03 & 0.02 \\
\hline Homa-IR & $4.64 \pm 1.38$ & $4.49 \pm 1.56$ & 0.49 & $4.22 \pm 1.43$ & $3.66 \pm 0.97$ & 0.025 & 0.045 \\
\hline Triglyceride $\mathrm{mg} / \mathrm{dL}$ ) & $137.7 \pm 66$ & $147 \pm 69.3$ & 0.42 & $147 \pm 69.4$ & $135 \pm 60.89$ & 0.27 & 0.65 \\
\hline IL-6 ( pg/ml) & $2.08 \pm 0.82$ & $1.85 \pm 0.59$ & 0.1 & $2.19 \pm 1.09$ & $1.58 \pm 1.06$ & 0.039 & 0.3 \\
\hline $\mathrm{TNF}-\alpha(\mathrm{pg} / \mathrm{ml})$ & $10.56 \pm 0.61$ & $10.57 \pm 0.68$ & 0.93 & $10.49 \pm 0.47$ & $10.33 \pm 0.66$ & 0.2 & 0.25 \\
\hline
\end{tabular}

Values are expressed as means \pm SD.

$* \mathrm{P}<0.05$ was considered as significant using Paired T-test.

$* * \mathrm{P}<0.05$ was considered as significant using Independent $\mathrm{T}$-test between the two groups post-intervention.

Table 4. Periodontal Status at baseline and post intervention.

\begin{tabular}{|c|c|c|c|c|c|}
\hline $\mathrm{PD}(\mathrm{mm})$ & Baseline & After 4 weeks & $\Delta$ & $P$-value * & $P$-value $* *$ \\
\hline Control group $(n=22)$ & $4.06 \pm 0.6$ & $3.38 \pm 0.5$ & $-0.6 \pm 0.47$ & $<0.001$ & $<0.001$ \\
\hline $\begin{array}{l}\text { Intervention group } \\
(n=21)\end{array}$ & $3.54 \pm 0.5$ & $2.35 \pm 0.6$ & $-1.1 \pm 0.58$ & $<0.001$ & \\
\hline
\end{tabular}

\section{Disc ussion}

In the present study, we found that receiving resveratrol supplementation for four weeks resulted in a significant decrease in insulin and insulin resistance (HOMA-IR), but there was no significant change in the serum levels of FBS. Furthermore, some inflammatory markers including IL6 were significantly reduced after using resveratrol supplementation (as a nutritional factor in adjunction with NST). It has previously been shown that serum level of some inflammatory markers including IL6 were high in patients with periodontal disease; such a reduction in these markers seen in this study may improve the infection occurred in periodontal status and affect blood glucose-dependent factors like insulin resistance in type 2 diabetic patients. Therefore, it is suggested that there may be a physiological relationship between controlling periodontal infection and diabetes mellitus. Nutritional factors ( such as anti-inflammatory and antioxidant nutritional compounds) are known to be important factors affecting periodontal disease, so it is expected that improving nutritional habits and diet may be useful in the treatment of periodontal disease (20,32). A study conducted by Sun et al. (2011) showed that treatment of periodontal disease in patients with diabetes led to controlling the blood sugar levels and a significant reduction in insulin resistance (33), which is in agreement with the findings of the present study. There are also other studies showing an improvement in blood sugar level after periodontal treatment $(34,35)$. Since lipopolysaccharide and inflammatory factors produced by bacteria may lead to insulin resistance, treatment of periodontal disease through elimination of these microorganisms can result in a reduction in these inflammatory markers and improve insulin sensitivity $(5,36)$. In agreement with our study, Brasnyo et al. showed that a four-week consumption of resveratrol significantly reduced insulin resistance in patients with type 2 diabetes (24). Mendez-del found that resveratrol supplementation in patients with metabolic syndrome resulted in a significant decrease in blood insulin area under the curve (AUC) but no significant change was seen in the curve for 
glucose level (37). Furthermore, one study by Crandall et al. showed that a four- week consumption of resveratrol supplement in patients with impaired glucose tolerance (IGT) improved insulin sensitivity and factors related to blood sugar after a meal, without a significant decrease in FBS (38). Similarly, in our study, no significant decrease was observed in fasting blood glucose levels. On the other hand, in a study conducted by Chen et al., there was a significant decrease in FBS after periodontal treatment in diabetic patients (39). Also, in a study by Debora et al., non-surgical periodontal treatment did not have a significant impact on glycemic control and FBS (40), which is consistent with our study. It is suggested that the beneficial effects of resveratrol are related to inactivation of $\mathrm{NF} / \mathrm{KB}$ stimulated by bacterial lipopolysaccharide in inflamed tissue and activation of SIRT and AMP kinase (31). Periodontal disease is an inflammatory process in which the leukocytes penetrate into the endothelium of inflamed periodontal tissues. It is believed that the main factor influencing inflammation is infection with oral pathogens. Bacterial Lipo-polysaccharides (LPS) induce the adhesion of leukocytes in periodontal tissue endothelium through the sticky molecules. Resveratrol significantly inhibited the bacterial lipopolysaccharides. The main mechanism suggested for inhibition of LPS by resveratrol is non-activation of the nuclear factor of $\mathrm{KB}(\mathrm{NF} / \mathrm{KB})$ (26). Sirtuin proteins (SIRT) are thought to improve mitochondrial function and energy limiting, there is a direct relationship between SIRT and the beneficial role of resveratrol including the improvement of glucose metabolism and insulin sensitivity. AMP kinase activated by SIRT has beneficial effects on the reduction of oxidative stress as the most important factor in insulin resistance, which results in improved insulin sensitivity and glycemic control $(21,41)$. Another possible mechanism is to relate resveratrol to sulfonylureas receptors and its effects on beta pancreas cells and insulin secretion (a mechanism that is similar to the effect of glibenclamide) (42). In Bhatt et al.'s study, receiving resveratrol supplementation in patients with diabetes mellitus for three months significantly reduced fasting plasma glucose and other risk factors related to glycemic control (27). In another study by Timmers et al., a 30-day consumption of resveratrol in healthy obese people resulted in a significant decrease in insulin, insulin resistance (HOMA-IR) and fasting blood glucose (30). These studies are inconsistent with the findings of the present study. Probably the length of intervention period in Bhatt's study and healthy subjects in Timmers's study may lead to such diversity in the FBS results. Therefore, further studies with longer duration of treatment are suggested in future. Patients with type 2 diabetes are affected by impaired glucose and lipid metabolism, which eventually leads to dyslipidemia in these patients (43). Also, in patients with diabetes mellitus and periodontal disease, there may be an increase in some inflammatory factors such as IL6 and TNF $\alpha$ that results in dyslipidemia and hypertriglyceridemia (44, 45). In one cohort study evaluating the relationship between metabolic syndrome and periodontal disease, Tu et al. showed that patients with periodontal disease have higher levels of blood triglycerides (14). In a study conducted by Mointaghavi et al., no significant decrease was observed in serum TG in diabetic patients after periodontal treatment (34). Furthermore, in a study by Crandall et al., no changes were observed in lipid profile including blood triglycerides (38). In the other study by Morten et al. in 2013 investigating the effect of receiving resveratrol supplements in healthy obese men, no significant changes were observed in lipid profile (46); these results are consistent with our study. Microorganisms associated with periodontal disease and host response to these microorganisms increase the production of inflammatory factors including IL6, TNF $\alpha$ and IL1B. These inflammatory markers are also involved in insulin resistance (45). In 2014, Nefumi et al. evaluated the effects of three weeks resveratrol supplementation on male Wistar rats with periodontal disease and observed an improvement in the inflammatory markers including IL6 and TNF $\alpha$ and a reduction in oxidative stress (47). In another study conducted by Rizzo et al., the effect of resveratrol supplementation and regulation of cytokine production on human periodontal ligament cells was investigated but no significant decrease was seen in the level of pre-inflammatory cytokines including IL1B, IL6 and TNF $\alpha$ (19). The results of these studies are inconsistent with our study. Moreover, in a study 
by Ghanim et al., after a six- week consumption of Polygonum cuspidatum containing resveratrol, a reduction was observed in the inflammatory markers of IL6, TNF $\alpha$ and CRP in healthy individuals (48). Tomé-Carneiro et al. also investigated the effect of one year enriched grape extract supplementation containing resveratrol on diabetic patients with hypertension and observed only a significant decrease in the serum levels of IL6 (49). This study is not consistent with the study conducted by Ghanim and Tomé-Carneiro. The duration of the intervention was greater than that of our study. In Morten's study, no significant reduction was observed in TNF $\alpha$ in healthy people after four weeks consumption of resveratrol supplementation (46). In a study by Chen et al., no significant reduction was observed in TNF $\alpha$ after periodontal treatments in patients with type 2 diabetes (39). Similarly, in our study, we found no significant reduction in $\mathrm{TNF} \alpha$ after four weeks consumption of resveratrol supplementation.

The link between various nutrients and systemic diseases has been established; however, relatively little work has been done in relating oral conditions with nutrition. Furthermore, to our knowledge, few studies have so far been conducted on the effects of nutritional intervention in adjunction with the nonsurgical periodontal treatment on periodontal status. Several in vitro studies support the beneficial effects of resveratrol on periodontal disease. Similarly, in the present study, there was a significant improvement in pocket depth post-intervention. To the best of our knowledge, there is no human study about the effects of resveratrol supplementation in patients with periodontitis. In one study carried out by Nafumi et al., the effects of resveratrol were investigated on Wistar male rats with periodontal disease, and it was shown that resveratrol reduced periodontitis and bone loss, increased antioxidant defense, and improved inflammatory factors; therefore, resveratrol supplementation was recommended for improving periodontal status (47). In a laboratory study in Korea, Joo Park et al. (2009) investigated the effect of resveratrol on lipopolysaccharide $P$. gingivalis, and suggested that resveratrol supplementation may help to remove periodontal pathogens (26).

There are some studies showing the beneficial effects of antioxidant or anti-inflammatory nutritional factors such as vitamin c, alpha-tocopherol, betacarotene, Q10, omega 3, green tea, probiotics and cranberry juice on periodontal status (50) Munozca et al. showed that receiving multi-vitamin nutritional supplement in periodontal patients resulted in a significant improvement in periodontal indices, especifically in pocket depth (51). Similarly, in a study by HeshamEl-Sharkawy et al., n-3 PUFA supplementation led to a significant decrease in pocket depth (52).

Conclusion: In the present study, resveratrol supplementation improved insulin resistance and periodontal status in diabetic patients with periodontal disease after four weeks. It is suggested that using resveratrol as a dietary supplement with antiinflammatory and anti-bacterial properties in adjunction with non-surgical periodontal treatments may be helpful in controlling the periodontal status followed by controlling some complications in diabetes mellitus. Therefore,it is suggested that using resveratrol supplementation and foods rich in resveratrol may be beneficial in diabetic patients with periodontal disease.

\section{Acknowledgement}

The authors express their thanks for Nutrition and Metabolic Disorders Research Center, and Research Center for Diabetes, Endocrinology and Metabolism clinic employees of Ahvaz University Golestan Hospital and Dental Clinic of Ahvaz Jundishapur University of Medical Sciences. This study is part of the M.Sc thesis of M.S. Hormoznejad.

\section{Financial disclosure}

The authors declared no financial interest.

\section{Funding/Support}

This work was financially supported by a grant (NRC-9304) from the Vice-Chancellor for Research Affairs of Ahvaz Jundishapur University of Medical Sciences.

\section{References}

1. Pranckeviciene A, Siudikiene J, Ostrauskas R, Machiulskiene V. Severity of periodontal disease in adult patients with diabetes mellitus in relation to the type of diabetes. Biomedical Papers. 2014;158(1):117-23

2. Amos AF, McCarty DJ, Zimmet P. The rising global burden of diabetes and its complications: estimates and projections to the year 2010. Diabetic Medicine. 1997;14(S5):S7-S85. 
3. Guariguata L, Whiting D, Hambleton I, Beagley J, Linnenkamp U, Shaw J. Global estimates of diabetes prevalence for 2013 and projections for 2035. Diabetes Research and Clinical Practice. 2014;103(2):137-49.

4. Dahiya P, Kamal R, Gupta R, Bhardwaj R, Chaudhary K, Kaur S. Reactive oxygen species in periodontitis. Journal of Indian Society of Periodontology. 2013;17(4):4.6-11

5. Grover HS, Luthra S. Molecular mechanisms involved in the bidirectional relationship between diabetes mellitus and periodontal disease. Journal of Indian Society of Periodontology. 2013;17(3):292-301.

6. Daniel R, Gokulanathan S, Shanmugasundaram N, Lakshmigandhan M, Kavin T. Diabetes and periodontal disease. Journal of Pharmacy \& Bioallied Sciences. 2012;4(Suppl 2):S280.

7. Emrich LJ, Shlossman M, Genco RJ. Periodontal disease in non-insulin-dependent diabetes mellitus. Journal of Periodontology .31-123: (2) 62:1991

8. Shlossman M, Knowler WC, Pettitt DJ, Genco RJ. Type 2 diabetes mellitus and periodontal disease. Journal of the American Dental Association (1939). 1990;121(4):532-6.

9. Offenbacher S. Periodontal diseases: pathogenesis. Annals of Periodontology. 1996;1(1):821-78.

10. Kara A, Akman S, Ozkanlar S, Tozoglu U, Kalkan Y, Canakci $\mathrm{CF}$, et al. Immune modulatory and antioxidant effects of melatonin in experimental periodontitis in rats. Free Radical Biology and Medicine. 2013;55:21-6.

11. Stanko P, Izakovicova Holla L. Bidirectional association between diabetes mellitus and inflammatory periodontal disease. A review. Biomedical papers of the Medical Faculty of the University Palacky, Olomouc, Czechoslovakia. 2014;158(1):35-8.

12. Taylor GW ,Burt BA, Becker MP, Genco RJ, Shlossman M, Knowler WC, et al. Severe periodontitis and risk for poor glycemic control in patients with non-insulindependent diabetes mellitus. Journal of Periodontology. 1996;67(10s):1085-93.

13. Negarto, C. A., Tarzia, O., Jovanovic, L., \& Chinellato, L. E. M. Periodontal disease and diabetes mellitus. Journal of Applied Oral Science, 2013, 21(1), 1.

14. Tu YK, D'Aiuto F, Lin HJ, Chen YW, Chien KL. Relationship between metabolic syndrome and diagnoses of periodontal diseases among participants in a large Taiwanese cohort. Journal of Clinical Periodontology. 2013;40(11):994-1000.

15. Kim EK, Lee SG, Choi YH, Won KC, Moon JS, Merchant AT, et al. Association between diabetes-related factors and clinical periodontal parameters in type-2 diabetes mellitus. BMC Oral Health. 2013;13:64.

16. Taylor GW, Borgnakke W. Periodontal disease: associations with diabetes, glycemic control and complications. Oral Diseases. 2008;14(3):191-203.

17. O'Connor DJ, Wong RW, Rabie AB. Resveratrol inhibits periodontal pathogens in vitro. Phytotherapy Research: PTR. 2011;25. (11);1.31-727

18. Long O, Ru-fan L. Effect of periodontal treatment on glycosylated hemoglobin levels in elderly patients with periodontal disease and type 2 diabetes. Chinese Medical Journal. 2011;124(19):3070-3.

19. Rizzo A, Bevilacqua N, Guida L, Annunziata M, Romano Carratelli C, Paolillo R. Effect of resveratrol and modulation of cytokine production on human periodontal ligament cells. Cytokine. 2012;60(1):197-204.

20. Zare Javid A, Seal CJ, Heasman P, Moynihan PJ. Impact of a customised dietary intervention on antioxidant status, dietary intakes and periodontal indices in patients with adult periodontitis. Journal of human nutrition and dietetics: the official journal of the British Dietetic Association.2014. Dec 1;27(6):523-532.

21. Vallianou NG, Evangelopoulos A, Kazazis C. Resveratrol and diabetes. The review of Diabetic Studies: RDS. 2013;10(4):236-42.

22. Szkudelska K, Szkudelski T. Resveratrol, obesity and diabetes. European Journal of Pharmacology. 2010;635 (1-3):1-8.

23. Fordham JB, Naqvi AR, Nares S. Leukocyte production of inflammatory mediators is inhibited by the antioxidants phloretin, silymarin, hesperetin, and resveratrol. Mediators of Inflammation. 2014; 2014:938712.

24. Brasnyo P, Molnar GA, Mohas M, Marko L, Laczy B, Cseh J, et al. Resveratrol improves insulin sensitivity, reduces oxidative stress and activates the Akt pathway in type 2 diabetic patients. The British Journal of Nutrition. 2011;106(3):383-9.

25. Uysal T, Gorgulu S, Yagci A, Karslioglu Y, Gunhan O, Sagdic D. Effect of resveratrol on bone formation in the expanded inter-premaxillary suture: Early bone changes. Orthodontics \& Craniofacial Research. 2011;14(2):80-7.

26. Park H-J, Jeong S-K, Kim S-R, Bae S-K, Kim W-S, Jin $\mathrm{S}-\mathrm{D}$, et al. Resveratrol inhibits Porphyromonas gingivalis lipopolysaccharide-induced endothelial adhesion molecule expression by suppressing NF- $\kappa \mathrm{B}$ activation. Archives of Pharmacal Research. 2009;32(4):583-91.

27. Bhatt JK, Thomas S, Nanjan MJ. Resveratrol supplementation improves glycemic control in type 2 diabetes mellitus. Nutrition Research. 2012;32(7):537-41.

28. Chachay VS, Kirkpatrick CM, Hickman IJ, Ferguson M, Prins JB, Martin JH. Resveratrol-pills to replace a healthy diet? British Journal of Clinical Pharmacology. 2011;72(1):27-38.

29. Yoshino J, Conte C, Fontana L, Mittendorfer B, Imai S-i, Schechtman KB, et al. Resveratrol supplementation does not improve metabolic function in nonobese women with normal glucose tolerance. Cell Metabolism. 2012;16(5):658-64.

30. Timmers S, Konings E, Bilet L, Houtkooper RH, van de Weijer T, Goossens GH, et al. Calorie restriction-like effects of 30 days of resveratrol supplementation on energy metabolism and metabolic profile in obese humans. Cell Metabolism. 2011;14(5):612-22.

31. Tamaki N, Cristina Orihuela-Campos R, Inagaki $\mathrm{Y}$, Fukui M, Nagata T, Ito HO. Resveratrol improves oxidative stress and prevents the progression of 
Ahmad Zare Javid, et al: Resveratrol supplementation in diabetic patients with periodontitis

periodontitis via the activation of the Sirt1/AMPK and the Nrf2/antioxidant defense pathways in a rat periodontitis model. Free Radical Biology \& Medicine. 2014; 222: 9-75

32. Gayoso-Diz P, Otero-González A, Rodriguez-Alvarez MX, Gude F, García F, De Francisco A, et al. Insulin resistance (HOMA-IR) cut-off values and the metabolic syndrome in a general adult population: effect of gender and age: EPIRCE cross-sectional study. BMC Endocrine Disorders. 2013;13(1):47.

33. Sun W-L, Chen L-L, Zhang S-Z, Wu Y-M, Ren Y-Z, Qin G-M. Inflammatory cytokines, adiponectin, insulin resistance and metabolic control after periodontal intervention in patients with type 2 diabetes and chronic periodontitis. Internal Medicine. 2011;50(15):1569-74.

34. Moeintaghavi A, Arab H, Bozorgnia Y, Kianoush K, Alizadeh M. Non-surgical periodontal therapy affects metabolic control in diabetics: a randomized controlled clinical trial. Australian Dental Journal. 2012;57(1):31-7.

35. Telgi RL, Tandon V, Tangade PS, Tirth A, Kumar S, Yadav V. Efficacy of nonsurgical periodontal therapy on glycaemic control in type II diabetic patients: a randomized controlled clinical trial. Journal of Periodontal \& Implant Science. 2013;43(4):177-82.

36. Simpson TC, Needleman I, Wild SH, Moles DR, Mills EJ. Treatment of periodontal disease for glycaemic control in people with diabetes. Australian Dental Journal. 2010;55(4):472-4.

37. Méndez-del Villar M, González-Ortiz M, MartínezAbundis E, Pérez-Rubio KG, Lizárraga-Valdez R. Effect of resveratrol administration on metabolic syndrome, insulin sensitivity, and insulin secretion. Metabolic Syndrome and Related Disorders. 2014;12(10):497-501.

38. Crandall JP, Oram V ,Trandafirescu G, Reid M, Kishore $\mathrm{P}$, Hawkins $\mathrm{M}$, et al. Pilot study of resveratrol in older adults with impaired glucose tolerance. The Journals of Gerontology Series A: Biological Sciences and Medical Sciences. 2012:glr235.

39. Chen L, Luo G, Xuan D, Wei B, Liu F, Li J, et al. Effects of non-surgical periodontal treatment on clinical response, serum inflammatory parameters, and metabolic control in patients with type 2 diabetes: A randomized study. Journal of Periodontology. 2012;83(4):435-43.

40. Rodrigues DC, Taba Jr M, Novaes Jr AB, Souza SL, Grisi MF. Effect of non-surgical periodontal therapy on glycemic control in patients with type 2 diabetes mellitus. Journal of Periodontology. 2003;74(9):1361-7.

41. Timmers S, Hesselink MK, Schrauwen P. Therapeutic potential of resveratrol in obesity and type 2 diabetes: new avenues for health benefits? Annals of the New York Academy of Sciences. 2013;1290(1):83-9.

42. Hambrock A, de Oliveira Franz CB, Hiller S, Grenz A, Ackermann S, Schulze DU, et al. Resveratrol binds to the sulfonylurea receptor (SUR) and induces apoptosis in a
SUR subtype-specific manner. Journal of Biological Chemistry. 2007;282(5):3347-56.

43. Iacopino AM, Cutler CW. Pathophysiological relationships between periodontitis and systemic disease: recent concepts involving serum lipids. Journal of Periodontology. 2000;71(8):1375-84.

44. Bastos AS, Graves DT, Loureiro APdM, Rossa Junior C, Abdalla DSP, Faulin TdES, et al. Lipid peroxidation is associated with the severity of periodontal disease and local inflammatory markers in patients with type 2 diabetes. The Journal of Clinical Endocrinology \& Metabolism. 2012;97(8):E1353-E62.

45. Wu Y-Y, Xiao E, Graves DT. Diabetes mellitus related bone metabolism and periodontal disease. International Journal of Oral Science. 2015.

46. Poulsen MM, Vestergaard PF, Clasen BF, Radko Y, Christensen LP, Stødkilde-Jørgensen H, et al. High-dose resveratrol supplementation in obese men an investigatorinitiated, randomized, placebo-controlled clinical trial of substrate metabolism, insulin sensitivity, and body composition. Diabetes. 2013;62(4):1186-95.

47. Tamaki N, Orihuela-Campos RC, Inagaki Y, Fukui M, Nagata T, Ito H-O. Resveratrol improves oxidative stress and prevents the progression of periodontitis via the activation of the Sirt1/AMPK and the Nrf2/antioxidant defense pathways in a rat periodontitis model. Free Radical Biology and Medicine. 2014;75:222-9.

48. Ghanim H, Sia CL, Abuaysheh S, Korzeniewski K, Patnaik P, Marumganti A, et al. An antiinflammatory and reactive oxygen species suppressive effects of an extract of Polygonum cuspidatum containing resveratrol. The Journal of Clinical Endocrinology \& Metabolism. 2010;95(9):E1-E8.

49. Tomé-Carneiro J, Larrosa M, Yánez-Gascón MJ, Dávalos A, Gil-Zamorano $\mathbf{J}$,Gonzálvez $\mathbf{M}$, et al. One-year supplementation with a grape extract containing resveratrol modulates inflammatory-related microRNAs and cytokines expression in peripheral blood mononuclear cells of type 2 diabetes and hypertensive patients with coronary artery disease. Pharmacological Research. 2013;72:69-82.

50. Soory M. Inflammatory mechanisms and redox status in periodontal and cardiometabolic diseases: effects of adjunctive nutritional antioxidants and statins. Infectious Disorders-Drug Targets (Formerly Current Drug TargetsInfectious Disorders). 2012;12(4):301-15.

51. Munoz C, Kiger R, Stephens J, Kim J, Wilson A. Effects of a nutritional supplement on periodontal status. Compendium of continuing education in dentistry (Jamesburg, NJ: 1995). 2001;22 32, 30, 8-425:(5)

52. El-Sharkawy H, Aboelsaad N, Eliwa M, Darweesh M, Alshahat M, Kantarci A, et al. Adjunctive treatment of chronic periodontitis with daily dietary supplementation with omega-3 Fatty acids and low-dose aspirin. Journal of Periodontology. 2010;81(11):1635-43. 